\title{
INTEGRATING LEARNING AND VISUALIZATION TECHNOLOGIES IN ORTHOPAEDICS - ESTABLISHING THE VIRTUAL ORTHOPAEDIC
EUROPEAN UNIVERSITY
}

\author{
S.GRANGE ${ }^{1,2,}$ CHRIS SETCHELL ${ }^{3}$, NEILL CAMPBELL ${ }^{3}$, G. WILLS ${ }^{4}$, \\ T.BUNKER ${ }^{1}$
}

1. Royal College of Surgeons of England, London, United Kingdom

2. Salisbury District Hospital, Salisbury, United Kingdom

3. Computer Science, University Of Bristol, United Kingdom

4. Electronics \& Computer Science, University Of Southampton, United Kingdom

\begin{abstract}
1. Purpose

Digital technologies offer a working environment for familiarisation with new surgical procedures and management of clinical case audit. Our aim is to provide a novel route for access to educational material that more closely resembles the working practice of the arthroscopist. This is to support higher surgical training and life long learning. The proof of concept has been the development of a shoulder arthroscopy simulation model as an interface for the surgical trainee to access multimedia based educational orthopaedic modules. This demonstrates a human-computer interface that more closely resembles the process of factual knowledge association during clinical procedures, moving toward the ultimate goal of seamless integration of knowledge repositories with clinical intervention augmenting thought and action.
\end{abstract}

\section{Methods}

To enhance access and control of these virtual workspaces an eXtensible Mark-up Language (XML) based interface has been developed. This links the educational environment of the Web Based Training (WBT) scheme and the clinical data collection from ongoing trials in Orthopaedics. The underlying Virtual Orthopaedic European University (VOEU) infrastructure encompasses the normal working environment of engineers and clinicians using computer assisted surgery tools adopting dedicated interactive media.

Adaptive hypermedia associates multiple hyperlinks to the simulation interface via frames in the video sequence of the video based surgical simulation environment. This associates the surgical experience of the trainee with the course material that they are covering. The material is prepared from actual patient operative video information, integrating the structured surgical course model with the multimedia educational orthopaedic modules, generated for the learning of shoulder surgery.

3. Results

An XML based interface enables users to communicate using material mediated for their specific needs allowing adaptive media based upon user experience and knowledge base. This combines declarative (factual) content with feedback from a clinical (procedural) case-based training and evaluation environment. By using the XML interface, we were able to cater for the different user hardware and software resources, media content and language of presentation, incorporated via the virtual environment simulation.

4. Conclusion

By constructing a framework based upon already established standards, we anticipate the applicability to other surgical disciplines. We also perceive this as a way of building patient specific arthroscopy datasets libraries (atlases) of pathologies. This will enable the development of networking computer architectures to assist the assimilation of multiple sources and media. The interface offers the opportunity to review data from computer assisted orthopaedic surgical systems embedded in an educational environment that is quantitative rather than descriptive. This will ultimately help the development of data repositories for mining, providing feedback upon clinical case management. Future work should focus upon the process of patient data collection and refinement of the image database production.

Keywords: surgical simulation, interface, vision based simulation, virtual university, orthopaedics, arthroscopy, adaptive hypermedia 


\section{INTRODUCTION}

\section{Who needs a Virtual University?}

There are three underlying motivators for the Information Society philosophy being applied to orthopaedics:

口 Constantly updating knowledge base for both procedural and declarative learning.

L Limited user time and computing expertise.

- Specialist knowledge base varies according to experience and application. With respect to the last of these, it is necessary to classify clinical knowledge into:

Essential. Part of the core curriculum relating to safety issues. Every trainee must be evaluated and demonstrate passable skills on every occasion. Should the trainee not be able to achieve a satisfactory standard then they will not be able to be awarded the relevant qualification. There is no negotiation with respect of these.

a Important. This is also part of the core curriculum. Although time is not formally available to test this, it will be included within the questions of the core modules. The varying ability to pass this will constitute the grade of performance.

a Further Reading. There is the need for a course to contain material that demonstrates various issues around the subject. In particular it adds background to assist with reflective learning. This does not need to be evaluated formally.

This background, blended with the variety of user experience that is encountered, necessitates virtual infrastructures for the training and evaluation of surgical performance. This requires reliable robust systems for the collection of data from both real-life and simulated environments. The data can then be used for both teaching of novice surgeons and the comparison of effective training techniques for different types of surgical intervention. We adopt the approach of the collecting data from four main fields. These are:

a Multimedia Educational Modules, which provide the declarative (factual) base of material for the education of the users - see figure 1 .

a A Virtual Classroom environment for exchange of views, and monitoring of progress.

- A Virtual Observatory for the collection of data from simulation systems and the actual intra-operative data collection

- Novel Modalities of Simulation ${ }^{(1: 2)}$ for the emulation of surgical procedures for training and experimentation focusing upon micro-surgery.

$\square$

Combining the above disciplines within

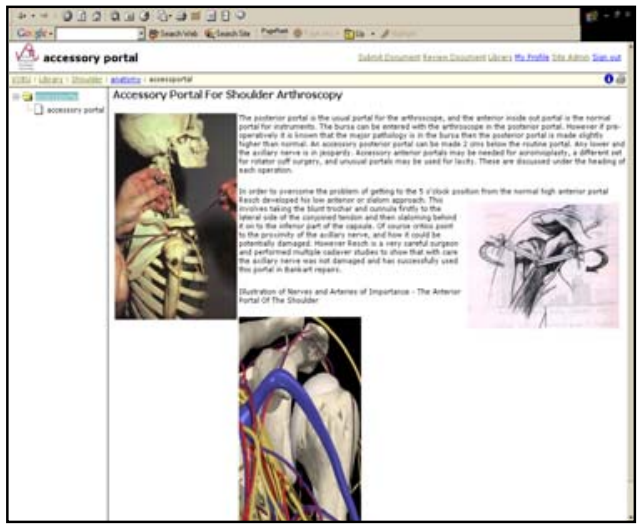

Figure 1- Multimedia Based Educational Orthopaedic Modules

one working environment, the virtual university infrastructure ${ }^{(3)}$ aims to meet the needs of clinicians to combining clinical, educational and research duties.

The Virtual Orthopaedic European University was specifically established to address the issues of data access, presentation and development of a structured learning environment for the training of novice surgeons.

\section{The End Users}


Surgeons are mobile well-educated individuals whose work demands excellent availability of educational material that is up to date and focused to their particular learning situation. This reflects the need for life long learning (refresher) material as well as the 'just in case' archives. New material is being collected constantly and this needs to be properly evaluated and integrated into the learning infrastructure appropriately.

This paper explains the process of data collection and retrieval. The system is being developed in a way that will support more sophisticated media including virtual environments.

The $\mathrm{XML}^{(4)}$ foundation presents the user with data in a form that is interpretable for their level of knowledge and experience. The system is fully scalable with respect to the number of collectable cases. It records a permanent archive of clinical procedures. Because of the sensitive nature of the material, the security issues are addressed at the server level and consent must be obtained from the patients for their case material to be included.

Part of the educational strategy employed relies upon the evolving university concept to allow for the updating and upgrading of educational material in light of new results, using analysis of incoming data from ongoing clinical trials for the evidence base. This acknowledges that a virtual university is a living infrastructure that evolves with time, due to changes both in its underlying philosophy and staff.

The construction of metadata standards for the core components, that abide by already approved standards such as the Dublin core, Learning Object Metadata and Information Management System (IMS), leads to a philosophy where components are 'living entities' whose survival within the university infrastructure relate to their relevance to users. Applicability (the ability to apply the technologies for other applications), and expandability ${ }^{(5 ; 6)}$ are vital characteristics of components in VOEU.

\section{METHODOLOGY}

\section{Can we develop a reliable knowledge grid for orthopaedics?}

Tim Berners-Lee originally led the WWW development at CERN in the 1980's. He established HTML for the structuring of physics related documents and files.

A philosophical rule of HTML was that it would convey the structure of a hypertext document. It does not however provide detail as to its presentation. It is a member of the broader group of standard generalized mark-up languages, but fails to provide other functions that are of value in maintaining its Generalisability $^{(7)}$. HTML does not for example; provide information that relates to the individual using the document. This can be introduced by allowing differing security levels access to parts of websites so that 'privileged' access can be granted. We do not however envisage that level of control of files as compatible with the role in healthcare, and could even be potentially unethical.

CERN provided a multiplatform, multicultural, microcosm in which to nurture the concepts that led to the evolution of the WWW. The pressures for information in medical applications are different and unique. The development of an evidence based culture combined with the progressive adoption of protocol driven services has led to the need to support such a philosophy with the tools to make appropriate peer reviewed information available directly to the clinician.

The approach to integrating the various components falls upon a graphical user interface referred to as a 'visual integrator'. This is primarily a web browser - HTML reader. Although add-ons are used for very specific functions, the majority of information must be presented to the user inside the frame of a standard HTML page, using scripts. Different components adopt different approaches to this - including the 
development of PERL, Java \& php server scripts, and the use of Microsoft SQL server Active Server Pages (ASPs) - see figure 2.
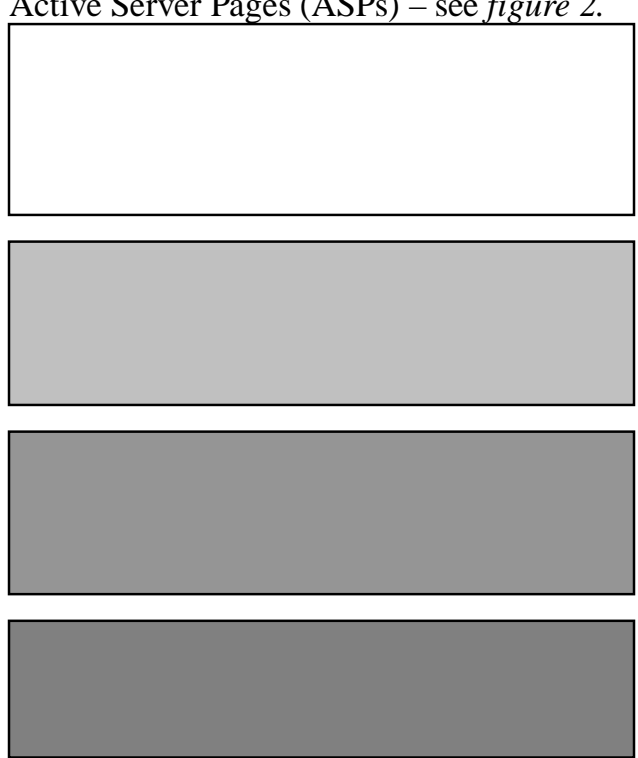

Figure 2. VOEU System Architecture Overview

To develop components that can be easily integrated, the web pages have a standard format adopting style sheets, allowing extension to other platforms with pervasive computing issues in mind. The media is supported by standard practices and is prescribed by a media report.

\section{Provision of customized multimedia educational modules to the user}

Surgery requires the operator to be versed in both practical (procedural) skills and factual (declarative) knowledge. The Virtual Orthopaedic European University is based upon the most recent technologies and standards for Virtual Learning Environment (VLE) development ${ }^{(8),(9)}$. The repository for educational packages is referred to as the Multimedia Educational Orthopaedic Modules (MEOM) component of VOEU. It is being built as an XML application based on Information Management System (IMS) Metadata and Content Packaging
Using IMS nomenclature, every educational item is treated as a resource whether it is a whole tutorial, a web page, multiple-choice question (MCQ) or an image. Each resource is described by Learning Object Meta-data (LOM) and can be used and reused in different context as the educational content developer sees fit. In the current version of VOEU the content can be organized in a varied but static way i.e. it is based on hierarchical structures of its governing content packaging Document Type Definition (DTD). Learner tracking will be developed allowing dynamic content building according to the individual's profile similar to the earlier Intelligent Tutoring Systems (ITS). The MEOM development concentrates on two areas: building an automated orthopaedic knowledge repository and serving it to the learner in the most educationally effective way. Figure 2 provides an overview:

Every educational resource, accompanied by its metadata will form an XML instance/document validated against the VOEUmetadata.dtd and stored in the repository. Content providers will be able to add resources automatically through an HTML form. Conversion of HTML to XML is handled by 'in house' software.

\section{Dynamic Review Journal (DRJ)}

Demonstrating the benefit of differing clinical approaches upon a small population of cases without considerable variation in outcome is difficult. In VOEU, a set of software tools offers an environment for accumulation of data, for mining to provide up to date reporting of various outcome measures set against the types of intervention. This forms the basis of the 'Dynamic Review Journal', which will focus upon:

a Medical evaluation of the submitted cases generating recommendations about the application of Information and Communication Technologies in orthopaedic surgery. 
a Technical and ergonomic evaluation to detect technical weaknesses in current Image Guided Orthopaedic Surgery (IGOS) systems. This will also serve to find potential fields of insufficient training in the usage of this technology.

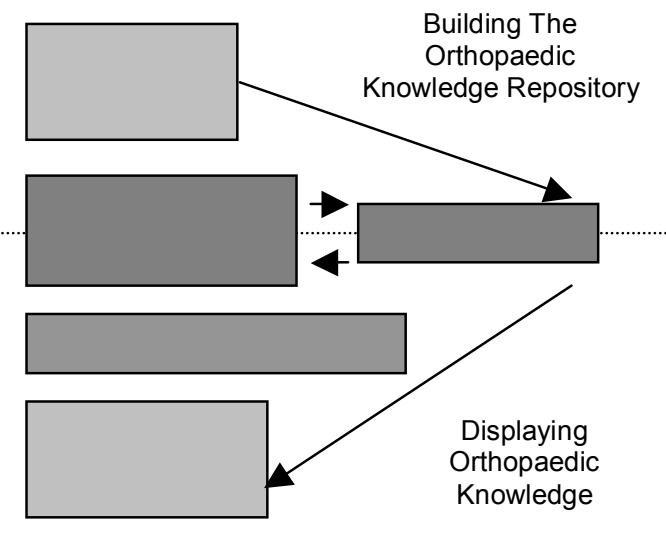

To assure quality, the cases that are output by the DRJ must be technically and medically validated. The technical validation is managed by constraints upon data collection. The medical validation is by the peer review of case results on an annual basis. The process for this is still in construction. A Static Review Journal (SRJ), which both stores and archives preprints and published articles - termed ePrints, is currently available.

It is likely that peer review will remain the most proficient indicator of performance during training for the foreseeable future.

Results are only credible if peer reviewed and are available for scrutiny. An infrastructure has been developed to support this by logging an individual's performance, in conjunction with the development of multimedia tools to guide the trainee.

\section{Personalized Libraries}

Gaining and retaining knowledge are personalized processes and need to be customizable to the level of the individual. The first stage is to match the user to a specific group who require core knowledge for their work. This should be culturally acceptable within the medical infrastructure and the society because the population accepts the fact that different 'grades' of staff have differing job descriptions requiring demonstration of differing levels of knowledge. The consortium adopts the perspective of the users. This involves educationalists, engineers and clinicians.

Users are likely to develop their own preferences for how the system is used. Data is therefore collected and stored centrally to allow the user to modify their preferences in accordance with the level of their expertise, matching the presentation of the data to the appropriate level of knowledge and experience.

\section{RESULTS}

Development of a virtual shoulder arthroscopy environment is already at the Beta testing stage - see figures $3 \& 4$.

One of the intrinsic advantages of designing, building and emulating minimal access surgery, is that the user is in effect already immersed within the appropriate operating environment, since they normally obtain a view of this indirectly via a monitor. The display system (interface) replicates the normal colour high definition (HDTV) monitor with which all the operators are familiar, avoiding cognitive dissonance.

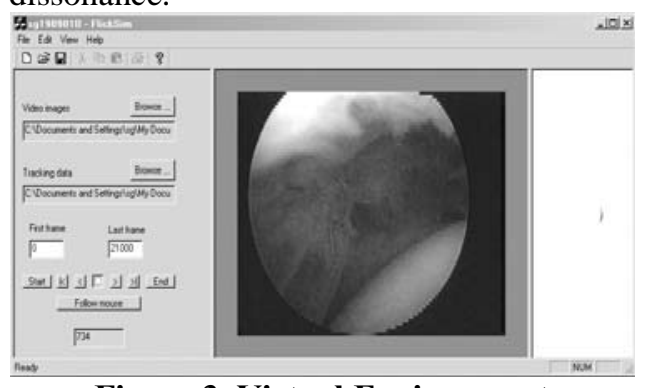

Figure 3. Virtual Environment Simulation Interface 
As Beta testing continues, it is possible to refine the applications so that they will be available to a wider audience, to adapt by adding new virtual environments and building a repository of cases that can be used both for paradigm (problem based) teaching and allow central collection of adequate data for both interim and long-term analysis of surgical progress.

Once the framework is in place and fully tested, it will be enhanced by the development of patient specific databases of information, including the potential to build patient specific virtual environments for the practicing of surgical procedures. This leads to the opportunity to create a range of different safe virtual testing environments for the design and evaluation of new surgical tools. The URL for this is available via the project website; http://voeu.ecs.soton.ac.uk/

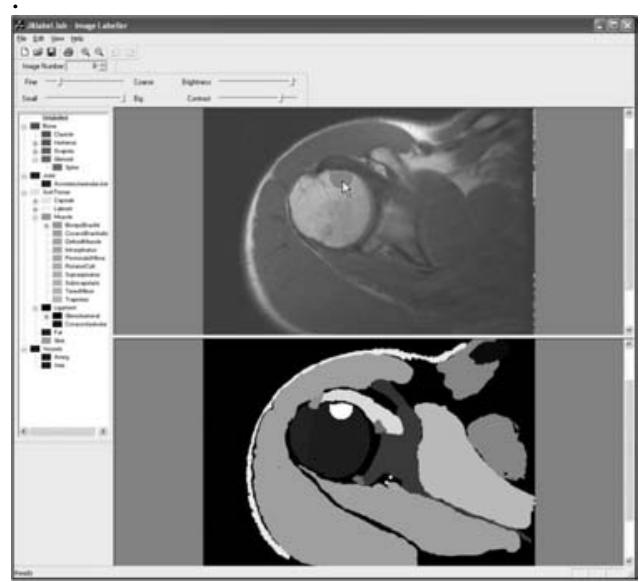

Figure 4. MRI Scan (Shoulder) Labeller DISCUSSION

The vocational training ${ }^{(11)}$ of anyone whose work involves clinically invasive procedures requires the best from that individual with regards to decision-making and psychomotor skills, thus the development and monitoring of these is vital. Ultimately simulator based skills training will become integrated within the framework of clinical governance (the quality assurance of clinical service delivery).

The methodology of previous task analysis for evaluation of surgical skills training was based upon the practical limits of what could be achieved with the available equipment at one site, and also the expert analysis of $\operatorname{tasks}^{(12),(13)}$. The results were inconclusive with a small number of trainees, as would be anticipated. It is therefore clear that a larger number of trial candidates are required. This is being addressed by the development of virtual observatories within the Virtual Orthopaedic European University ${ }^{(14)}$.

\section{Who benefits from a Virtual University?}

The appropriate knowledge category depends upon the clinical role that the individual plays and their stage of training. It is thus the responsibility of course convenors to ascribe various XML tags to learning objects to match the syllabus for the grade of the trainee. This allows for focused presentation of material to the user group. This is achieved by providing 'Look-up' tables for each grade of trainee.Improved functionality results in increased complexity of the interface by using a derivative of Structured Generalized Mark-up Language (SGML). The XML abides by standards being evolved for this domain.

by IEEE and where possible, the international organization, Health Level Seven (www.HL7.org). This will ensure greater compatibility between the interfaces that are used for the control of surgical procedural trainers (simulators).

There are potential pitfalls though. The most significant of these is security. The system is stable, and likely to display good survivability characteristics ${ }^{(15)}$ though the open nature of the network, and its potential application for providing communication portals between the machines, can provide a vehicle for corrupting software (viruses).

\section{CONCLUSION}


Virtual University infrastructures are no longer optional. They are essential. From an acorn, great oak trees grow, and so too with the university (XML) infrastructures, since these tools will greatly assist data mining and thus all areas of future work within the environment. These tools should be free at the point of delivery for users who are likely to be granted access via institutions and societies.

VOEU has established DTDs and these may ultimately be integrated into an educational schema though this is not currently planned.

VOEU's role as a knowledge construction tool, integrating concept mapping with supporting data repositories offers a powerful base to support research, clinical and educational tasks. Analysis via the dynamic review journal will update intervention records automatically with the mainstream orthopaedic community being able to review results personally and evaluate them in a digital peer reviewed framework.

XML plays a significant role in system integration. This allows different simulation systems, each with the capabilities to provide training and evaluation in specific surgical sub-skills, to contribute data for evaluation. It will facilitate statistical analysis of suitably sized populations of surgeons, saving effort by providing different grades of user with the appropriate information that they require.

The relevant information is included in the syllabus of the Educational Modules. This brings the university to life as it evolves with the collection of reviewed data. The database will host the relevant learning cases collected clinically that will then be used as test cases for validation purposes of surgical simulators. This will attempt to resolve the self-referential paradox, that; "One cannot properly assess the performance without first knowing the performance of the population who are testing it". Simulated virtual environments can then be designed into the interface as portals.
The data of simulator performance will progressively improve in accuracy and ultimately provide a system of assessment with the underlying foundation of a large population of surgeons, due to the use of distributed computing architecture to develop this important database.

The aim of future work is to ensure that these devices assure compatibility between the intra-operative tools developed for the training and microsurgical operating environments plus the extension of the archive of cases for testing of new tools plus offering pervasive viewing of clinical visual data. The simulations will be linked via the adaptive hyper media system to act as portals spiced with the particular links requested by the user. This helps to integrate visualization process with the learning content

\section{Acknowledgements}

The European Commission supports this project (Project VOEU, IST-1999-13079, Information Society Technology Program).

The authors wish to recognise the effort of the Intelligence, Agents, and Multimedia Group (IAM) at the University of Southampton, United Kingdom - Pr. Les Carr and Mr Guillermo Power, for their work upon the Dynamic Review Journal development based upon Open Archives Initiative (OAI) protocols. 


\section{BIBLIOGRAPHY}

[1] Grange S., Bunker T., Cooper J. Networking virtual reality for shoulder arthroscopy. British Journal of Healthcare Computing 13[10], 2628. 1996.

[2] Exeter Virtual Worlds Shoulder Arthroscopy Simulator. London: Brunel Univ Press, 1997.

[3] Hazemi R, Hailes S. Reinventing the Academy. In: Wilbur S, Hazemi R, Hailes S, editors. The Digital University. London: Springer, 1998: 7-24.

[4] T.Bray, C.M.Sperberg-McQueen, J.Paoli, E.Maler. Extensible Markup Language [XML] 1.0. W3.org 2. 2000.

[5] Wactlar H.D., Christel M.G., Gong Y., Hauptmann A.G. Lessons Learned from Building a Terabyte Digital Video Server. Computer 82[2], 66-73. 1999.
[6] Witten W.I., Mc Nah R.J., Jones S., Apperley M., Bainbridge D., Cunningham S.J. Managing Complexity in a Distributed Digital Library. Computer 32[2], 74-79. 1999.

[7] Tim Berners-Lee, Mark Fischetti. A solution: Hypertext. Weaving the web. London: TEXERE Publishing Ltd, 1999: 229-252.

[8] Linda Harasim. A Framework for Online Learning: The Virtual-U. Computer 32[9], 44-49. 1999.

[9] Schatz B., Chen H. Digital Libraries Technological Advances and Social Impacts. Computer 82[2], 45-50. 1999.

[10] IMSContent Information Model. 2001

http://www.imsproject.org/content/pa ckaging/cpinfo10.html.

[11] Dearing R. The Dearing Report. Dearing R, editor. 1, Ch13. 1997.
Natioonal Committee of Inquiry into Higher Education [NCIHE].

[12] Bunker T.D., Schranz P.S. The art of diagnosis in the mystery shoulder. Clinical Challenges in Orthopaedics: The shoulder. Oxford: ISIS Medical Media, 1998: 1-23.

[13] Preece J. What is HCI? HumanComputer interaction. Harlow: Addison-Wesley, 1998: 3-27.

[14] VOEU Consortium. Virtual Orthopaedic European University. IST - 13079 - 1999 . 2001.

[15] Ellison R.J., Fisher D.A., Linger R.C., Lipson H.F., Longstaff T.A., Mead N.A. Survivability: Protecting your critical systems. IEEE Internet Computing 3[6], 55-63. 1999. 